\section{La aplicación del conocimiento}

\section{Doctor}

\section{Eugenio Matijasevic \\ Editor General}

\section{Acta Médica Colombiana}

Apreciado doctor Matijasevic:

Muy importante y bien documentado su Editorial, "La aplicación del conocimiento" (1), en el cual analiza un estudio publicado recientemente en New England Journal of Medicine sobre el uso de oxígeno en pacientes con EPOC e hipoxemia moderada (2) y su dudosa aplicabilidad a grandes alturas, y hace énfasis en la necesidad de investigar localmente acerca de problemas cotidianos en salud. El ejemplo es muy apropiado puesto que los criterios de oxigenoterapia a largo plazo (OLP) en pacientes con EPOC fueron definidos a nivel del mar y cerca del $20 \%$ de la población colombiana reside por encima de los 2.500 metros. Al respecto, aportamos la siguiente información:

1. Una de las clasificaciones de altitud más recomendadas (3) define grandes alturas como aquéllas situadas entre 2.500 y 3.500 metros sobre el nivel del mar. Bogotá, a 2.640 metros, es la ciudad más poblada del mundo situada a gran altura (México D.F. está a $2.240 \mathrm{~m}$ ).

2. El estudio sobre valores normales de gases arteriales en adultos sanos en Bogotá $(n=374)$ (4), en preparación para publicación, muestra que una saturación de oxígeno entre 89 y $93 \%$, definida como hipoxemia moderada en el estudio analizado en su Editorial $(1,2)$, está en rangos de normalidad para la altitud de Bogotá.

3. Como usted lo plantea (1), se desconoce si los criterios de OLP en EPOC definidos a nivel del mar son apropiados a gran altura. Basados en el estudio sobre gases arteriales normales en Bogotá (4) y en la observación de que un grupo significativo de pacientes con EPOC, residentes a esta altura, con presión arterial de oxígeno entre 50 y $55 \mathrm{mmHg}$ no exhiben signos de hipoxia crónica, particu- larmente hipertensión pulmonar, planteamos un estudio experimental multicéntrico, coordinado desde la Fundación Neumológica Colombiana, para evaluar si se puede hacer un ajuste (punto de corte más bajo) de los criterios de OLP a gran altura $(\geq 2.500-\leq 3.500 \mathrm{~m})$. El estudio fue aprobado a comienzos de 2016 por COLCIENCIAS (335972553295) y se encuentra registrado en Clinical Trials (www.clinicaltrials .gov) (NCT03020212). Tendrá una duración de 36 meses y se espera que a comienzos de la siguiente década se tengan resultados que den respuesta a la pregunta planteada en su Editorial.

4. Como información relacionada, un análisis colaborativo (BOLD, PLATINO, EPISCAN y PREPOCOL) recientemente publicado, comprueba que no hay asociación entre altitud y prevalencia de EPOC (5) como lo había sugerido el estudio PREPOCOL previamente.

Cordialmente,

Carlos A. Torres Duque Director, Departamento de Investigación, Fundación Neumológica Colombiana Coordinador, Programa de Especialización en Neumología, Universidad de La Sabana. Profesor, Universidades de La Sabana y El Rosario, Bogotá

María José Pareja Zabala Médica, Departamento de Investigación, Fundación Neumológica Colombiana, Estudiante de maestría en Epidemiología, Bogotá

\section{Bibliografía}

1. Matijasevic E. La aplicación del conocimiento. Acta Med Colomb 2017;42:1-11.

2. The long-term oxygen treatment trial research group. A randomized trial of longterm oxygen for COPD with moderate desaturation. N Engl J Med 2016;375:16171627.

3. Barry PW, Pollard AJ.Altitude illness. BMJ (Clinical research ed) 2003;326:915919.

4. Maldonado D, Gonzalez-Garcia M, Barrero M, Casas A, Torres-Duque C. Reference values for arterial blood gases at an altitude of 2640 meters. Am J Respir Crit Care Med 2013;187:A4852.

5. Horner A, Soriano J, Puhan M, Studnicka M, Kaiser B, Vanfleteren E, Gnatiuc L, Burney P, Miravitlles M, García-Rio F, Ancochea J, Menezes A, PerezPadilla R, Montes de Oca M, Torres-Duque C, Caballero A, González-García M, Buist S, Flamm M, Lamprecht B. Altitude and COPD prevalence: Analysis of the prepocol-platino-bold-epi-scan study. Respiratory Research 2017;18. 


\section{Respuesta del Editor}

Estimados Doctores

Carlos A. Torres Duque y María José Pareja Zabala:

Gracias por su comentario al Editorial La Aplicación del Conocimiento (1), gracias también por los datos que aportan al problema perenne de la salud en las alturas, en especial el énfasis que hacen en que, entre las ciudades situadas por encima de 2500 metros sobre el nivel del mar (msnm), Bogotá es la más poblada del mundo; y felicitaciones por los trabajos de investigación que están realizando.

Su estudio de próxima publicación sobre valores normales de gases arteriales en adultos sanos en Bogotá (2) es precisamente la clase de investigaciones a la que hice referencia en el mencionado Editorial al hablar de ciencia local: conocimientos nuestros, obtenidos por nuestros investigadores y con utilidad inmediata para tomar decisiones clínicas correctas con respecto nuestros pacientes. Sin embargo, debo resaltar que, a diferencia de los ejemplos de ciencia local que aduje sobre cómo llegan a hemodiálisis nuestros pacientes con insuficiencia renal crónica (más de $90 \%$ a través del servicio de urgencias) $(3,4)$, la investigación realizada por ustedes (en la medida en que no está implícita la idiosincrasia de nuestro sistema de salud, ni la burocracia que lo corroe, ni el afán de lucro de los dueños de la intermediación financiera, sino que depende exclusivamente de las características fisiológicas de una muestra de la población que reside a gran altura), posee un carácter más allá de lo local: sus resultados son de utilidad universal en el amplio campo, aún a medias explorado, de la fisiología de la adaptación de nuestra especie a la altura (5), y esto es así incluso aunque dichos resultados no puedan ser extrapolados de manera directa a los pobladores de las alturas en los otros países andinos o en los valles del eje neovolcánico de México o en la meseta Tibetana o en las tierras altas de Nepal, Bután, Yemen, Afganistán, Eritrea y Etiopía.

Con respecto al estudio multicéntrico que han planeado e iniciado, dirigido a evaluar si en alturas entre los $2500 \mathrm{y}$ los $3500 \mathrm{msnm}$ se puede establecer un punto de corte más bajo en los criterios de requerimiento de oxígenoterapia a largo plazo, quiero insistir en que es éste el tipo de estudios que Colombia y América Latina requieren; estudios dirigidos a responder preguntas planteadas por la mirada del investigador puesta en nuestros problemas autóctonos de salud; problemas tan propios que son en cierta medida exclusivos, condicionados como estamos por una geografía específica, por unas características socioculturales aún más específicas y por unas variaciones y derivas genéticas más precisas aún (parece ser que los habitantes de la meseta Tibetana han seguido un camino de adaptación a la altura genéticamente diferente a los de las alturas andinas) (6). He podido consultar el protocolo que remitieron a ClinicalTrials.gov (7) y observo con agrado que han elegido unos criterios de valoración (la traducción al castellano que prefiero y prefieren muchos en lugar del anglicismo end-point) (8) que se apartan del que, en general, se ha considerado el criterio de valoración (end-point) absoluto: la mortalidad. En su lugar, han elegido criterios de valoración subrogados (desarrollo de hipertensión pulmonar determinada mediante ecocardiograma, aparición de poliglobulia, modificación del VEF1 y la CVF, número de exacerbaciones, cambios en la calidad de vida mediante el St. George Respiratory Questionnaire, prueba de minimental y prueba de marcha de seis minutos) que, sin embargo, permitirán responder a cabalidad la pregunta inicial, que podría parafrasearse en algo así como “¿Es la oxigenoterapia a largo plazo benéfica o es ineficaz en los pacientes con EPOC con desaturación moderada residentes a alturas entre 2500 y 3500 msnm?". Esta afirmación merece una pequeña digresión: el efecto de una intervención ("ser benéfica" o de "ser ineficaz") mencionado en la paráfrasis de la pregunta inicial, no se refiere únicamente a la mortalidad; además de la muerte son muchas otras las situaciones clínicas en EPOC que tanto pacientes como médicos deseamos evitar, por ejemplo: llegar a requerir trasplante pulmonar, empeoramiento de la disnea de esfuerzo o de la fatiga muscular, tener que hospitalizarse, limitación en las actividades de la vida diaria (9); de tal manera que, mientras la intervención no acorte el tiempo hasta la muerte (y hay bastante evidencia de que la oxigenoterapia a largo plazo tiene exactamente el efecto contrario), no hay razón para no utilizar criterios de valoración subrogados. Quise insistir en este punto porque sé que son muchas las voces que se preguntarán por qué entre los criterios de valoración elegidos sólo se encuentran criterios de valoración subrogados y no figura la mortalidad. En síntesis, somos muchos quienes esperamos ansiosos los resultados de este estudio, resultados que podrían tener también aplicación en otras zonas de la geografía mundial hechas las adaptaciones necesarias o, mejor aún, replicando el estudio en dichas regiones.

Felicitaciones de nuevo.

\section{Eugenio Matijasevic}

Editor General de Acta Médica Colombiana

\section{Referencias}

1. Matijasevic E. La aplicación del conocimiento. Acta Med Colomb 2017; 42 (1) $1-11$.

2. Maldonado D, Gonzalez-Garcia M, Barrero M, Casas A, Torres-Duque C. Reference values for arterial blood gases at an altitude of 2640 meters. Am J Respir Crit Care Med 2013; 187: A4852.

3. Coronado CY, Lombo JC, Correa I, Quintero N. Características clínicas y demográficas de los pacientes incidentes en diálisis crónica y su relación con el ingreso programado a diálisis. Acta Med Colomb 2013; 38 (3): 138-142.

4. Ortega M, Martínez JA, Gamarra G. Mortalidad en los pacientes con falla renal crónica durante los primeros 90 días de terapia con hemodiálisis. Acta Médica Colomb 2006; 31 (1):13-9.

5. Swenson ER, Bärtsch P. High Altitude: Human Adaptation to Hypoxia. New York: Springer; 2014: 496 pp. 
6. Bigham AW, Wilson MJ, Julian CG, et al. Andean and Tibetan patterns of adaptation to high altitude. Am J Hum Biol 2013; 25 (2):190-7.

7. Torres-Duque CA et al. Long-term Oxygen Therapy in Patients With Chronic Obstructive Pulmonary Disease (COPD) Who Live at High Altitude (2640 Meters Above Sea Level): Evaluation of a Proposal for Adjusting Criteria. Consultado el 20 de septiembre de 2017. Disponible en: https://clinicaltrials.gov/ct2/show/NC
T03020212? cond $=$ COPD\&intr $=$ Oxygen \&cntry $1=$ SA $\% 3$ ACO\&rank $=1$

8. Navarro FA, González J. Palabras y expresiones inglesas de traducción difícil o engañosa en investigación clínica, bioestadística y «medicina basada en la evidencia». Emergencias 2014; 26 (5): 375-92.

9. Snow JL, Kawut SM. Surrogate end points in pulmonary arterial hypertension: assessing the response to therapy. Clin Chest Med 2007; 28 (1): 75-89. 